\title{
A Low Complexity Interference Cancellation Technique for Multi-User DS-CDMA Communications
}

\author{
Wen-Bin Yang and Kamran Sayrafian-Pour \\ Information Technology Laboratory \\ National Institute of Standards and Technology
}

\begin{abstract}
A low complexity Parallel Interference Cancellation (PIC) technique is proposed to suppress Multi-Access Interference (MAI) and minimize near-far effect for multi-user communication using Direct-Sequence Code Division Multiple Access (DS-CDMA). Interference signal is estimated using a relationship between cyclic correlation of the received signal and user's code of the interferer without requiring any knowledge of the channel condition. The user's codes for the multi-user DSCDMA communication are constructed by using a set of $\mathrm{m}$ sequences. The cyclic correlation is performed using Fast WalshHadamard Transform (FWHT) which exhibits low computational complexity, i.e., it does not require any multiplication/division operations. In addition to low complexity, the proposed technique does not require complicated channel condition estimation and has no convergence issues. The uncoded Bit Error Rate (BER) performance of the proposed interference cancellation is calculated and compared with the conventional one over three different types of channels: AWGN, slow Rayleigh fading, and multipath channels.
\end{abstract}

Index Terms - Interference cancellation, Direct-sequence code division multiple access, Fast Walsh-Hadamard transformation

\section{INTRODUCTION}

$I^{N}$ $\mathrm{N}$ a Direct-Sequence Code Division Multiple Access (DSCDMA) system, the desired signal of a user is detected and decoded against interference by using the de-spreading feature of a code sequence corresponding to that user. All other users in this system are simply considered as Multi-Access Interference (MAI). The performance could be degraded when total interference increases due to the increasing number of users. Even when the number of users is not large; the received signal from nearby or high-powered interfering users may be too high; resulting in overwhelming the signal from the desired user. This is called the near-far effect which causes performance degradation in detecting or decoding the desired signal. To minimize the near-far effect, an efficient power control mechanism is usually required. However, implementing such mechanisms could be very complicated in fast fading environments or when the users are highly mobile. In such situations, controlling the transmit power based on the channel conditions in order to keep the received signal at a desired level could be very challenging. Another example is in underwater acoustic communication systems where long propagation delays make effective power control extremely difficult. Therefore, to overcome the power control problem in such tough environments, interference cancellation technique will be necessary [1]. In general, an interference cancellation technique not only minimizes the near-far effect but also reduces the MAI. Ultimately, the reduction of interference effect improves the system capacity; and therefore, allows more simultaneous users in a given DS-CDMA network.

There are two main disadvantages in the previously proposed interference cancellation techniques. First is the high complexity of the receiver which makes the implementation of such techniques impractical unless the number of users is very small [2-3]. Second disadvantage is that some interference cancellation schemes require perfect knowledge of the channel condition (such as attenuation, phase and delay) between each of the interferers and the receiver [4-6]. Obtaining accurate estimates of the channel condition is not only difficult but also requires some extra bandwidth. In this paper, we propose an interference cancellation technique that has a low complexity and does not require channel estimation using extra bandwidth. The technique may be applied to various DSCDMA networks such as cellular networks or wireless local and personal area networks.

The remainder of this paper is organized as follows. Section II provides a description of the DS-CDMA system. The proposed method of interference estimation and cancellation is presented in section III. Section IV describes the Fast WalshHadamard Transformation (FWHT) for implementation of interference estimation. Finally, simulation results and conclusions are discussed in Sections V and VI respectively.

\section{SYSTEM DESCRIPTION}

In a multi-user DS-CDMA network, $K$ users could be simultaneously transmitting in the same frequency band. The $k^{\text {th }}$ user is assigned a spreading code sequence, $c_{l}^{k}, l=0,1, \ldots, L-1$, with spreading factor $L$. The transmitted baseband signal from the $k^{\text {th }}$ user is given by

$$
S^{k}(t)=\sum_{i} d_{i}^{k} C^{k}\left(t-i T_{s}\right)
$$

where $d_{i}^{k}$ is the $i^{\text {th }}$ symbol transmitted by user $k$, 
$C^{k}(t)=\sum_{l=0}^{L-1} c_{l}^{k} g\left(t-l T_{c}\right), T_{c}$ is the chip duration, $T_{s}$ is a symbol duration $\left(T_{s}=L^{*} T_{c}\right)$ and $g(t)$ is a unit rectangular pulse centered at 0 with width $T_{c}$. Thus, for a specific receiver, the received signal from the $k^{\text {th }}$ user during the $i^{\text {th }}$ symbol period is

$$
r_{i}^{k}(t)=s_{i}^{k} d_{i}^{k} C^{k}(t) \otimes h^{k}(t), \text { for } i T_{s} \leq t<(i+1) T_{s}
$$

where $s_{i}^{k}$ is the received signal strength, $h^{k}(t)$ is the channel impulse response from the $k^{\text {th }}$ transmitter to the given receiver and $\otimes$ denotes the convolution operator. The channel impulse response can be modeled as shown in Eq. (3)

$$
h(t, \xi)=\sum_{l=0}^{L-1} \alpha_{l}(t) \delta\left(t-\xi_{l}\right) e^{j \theta_{l}(t)}
$$

where $\alpha_{l}(t)$ and $\theta_{l}(t)$ are amplitude and phase of a channel impulse response and $\xi_{l}$ is the time delay corresponding to $l^{\text {th }}$ chip duration. The total received baseband signal from all users can be described by Eq. (4)

$$
r_{i}(t)=\sum_{k=1}^{K} r_{i}^{k}\left(t-\tau_{k}\right)+n(t)
$$

where $\tau_{k}$ is the time delay with respect to a reference time and $n(t)$ is the Additive White Gaussian Noise (AWGN). For simplicity, we ignore the noise at this time and assume that the receiver is interested in decoding the signal from user 1 and $\tau_{1}=0$. To extract the $1^{\text {st }}$ user's signal from the total received signal, one can use cross-correlation between the received baseband signal and the code sequence of user 1 as shown in Eq. (5)

$$
\begin{aligned}
S_{i}^{1} & =C^{1}(t) \bullet r_{i}(t)=s_{i}^{1} d_{i}^{1} \rho^{1,1}(t) \otimes h^{1}(t) \\
& +\sum_{k=2}^{K} s_{i}^{k} d_{i}^{k} \rho^{1, k}\left(t, \tau_{k}\right) \otimes h^{k}\left(t-\tau_{k}\right)
\end{aligned}
$$

where $\rho^{1, k}\left(t, \tau_{k}\right)=C^{1}(t) \bullet C^{k}\left(t-\tau_{k}\right)$ is the cross-correlation function of the $1^{\text {st }}$ code sequence with the $k^{\text {th }}$ code sequence and $\bullet$ denotes the cross-correlation operator.

The first term on the right side of equation (5) represents the desired signal and the second term is the interference. Now, if the code sequence of user 1 is orthogonal to the spreading code sequences of other users with any time-shift (i.e. $\rho^{1, k}\left(t, \tau_{k}\right)=0$ when $\left.k \neq 1\right)$, then the effect of interference from users 2 to $K$ in Eq. (3) becomes zero. But, in reality, $\rho^{1, k}\left(t, \tau_{k}\right) \neq 0$ when $k \neq 1$, and the interference will be a nonzero component. If $s_{i}^{k} \gg s_{i}^{1}$ for any $k$, then this interference becomes significant. In general, even compatible levels of interference cause degradation in performance and reduction in system capacity when there is no interference cancellation.

In this paper, we consider using $\mathrm{m}$-sequences as the spreading codes. The auto-correlation function of an $\mathrm{m}$ sequence, with $m$ being an integer, is close to the desirable impulse function. Furthermore, the cross-correlation between any two m-sequences is very small as they are almost mutually orthogonal. Therefore, they are an ideal candidate to implement an interference cancellation scheme. Based on this orthogonality property, interference can be estimated using the cross-correlation of the received signal and the m-sequence of the interferer. In [7-8], an interesting relationship between $\mathrm{m}$ sequences and the Hadamard Transform has been discussed. This relationship allows for fast computation of the crosscorrelation (as well as its inverse) of the received signal and the m-sequence of the interferer. This is referred to as Fast Walsh-Hadamard Transform (FWHT). In the following two sections, we will describe how to use correlation to estimate and suppress interference; and then, how to use the lowcomplexity FWHT to implement the interference cancellation technique.

\section{INTERFERENCE ESTIMATION AND CANCELLATION}

Let the discrete received baseband signal at $i^{\text {th }}$ symbol duration be $\underline{r}^{i}=\left[r^{i}(1), r^{i}(2), r^{i}(3), \ldots r^{i}(m L)\right]^{t}$, where $m$ is the number of samples per chip and the superscript $t$ represents the transpose operation. This vector can also be reshaped to an $(m \times L)$ matrix where the $j^{\text {th }}$ row vector of the matrix is $\underline{r}_{j}^{i}=\left[r^{i}(j), r^{i}(m+j), r^{i}(2 m+j), \ldots r^{i}((L-1) m+j)\right]^{t}$,

$$
j=1,2, \ldots, m \text {. }
$$

Now, consider the interferer to be the $k^{\text {th }}$ user with code sequence $\underline{c}^{k}=\left[c_{0}^{k}, c_{1}^{k}, \ldots c_{L-1}^{k}\right]$. Each of the row vectors is correlated with the user code as below.

$$
\underline{\hat{r}}_{j}^{i, k}=\underline{c}^{k} \bullet \underline{r}_{j}^{i}
$$

The correlation output can be well-approximated by a cyclic cross-correlation since the m-sequence has a good autocorrelation property. The cyclic cross-correlation can be written in matrix form as:

$$
\underline{\hat{r}}_{j}^{i, k}=M_{L}^{k} \cdot \underline{r}_{j}^{i}
$$

where $M_{L}^{k}$ is a circulant m-sequence matrix for the given user code $\underline{c}^{k}$ as shown below. 


$$
M_{L}^{k}=\left[\begin{array}{ccccc}
c_{0}^{k} & c_{1}^{k} & \cdots & c_{L-2}^{k} & c_{L-1}^{k} \\
c_{L-1}^{k} & c_{0}^{k} & \cdots & c_{L-3}^{k} & c_{L-2}^{k} \\
\vdots & \vdots & \ddots & \vdots & \vdots \\
c_{2}^{k} & c_{3}^{k} & \cdots & c_{0}^{k} & c_{1}^{k} \\
c_{1}^{k} & c_{2}^{k} & \cdots & c_{L-1}^{k} & c_{0}^{k}
\end{array}\right]
$$

The correlation output contains two components, the signal from user $k$ and a noise-like component that is due to all other users. As the value of this second component is usually below the signal of user $k$, one can define a threshold $\eta$ to remove it from $\underline{\hat{r}}_{j}^{i, k}$ as indicated in Eq. (9)

$$
\underline{\underline{r}}_{j}^{i, k}(l)=\left\{\begin{array}{cc}
\hat{\underline{r}}_{j}^{i, k}(l) & \text { if } \hat{\underline{r}}_{j}^{i, k}(l) \geq r_{p} / \eta \\
0 & \text { elsewhere }
\end{array} \text {, for } l=0,1, \ldots, L-1,(9)\right.
$$

where $r_{p}=\max _{l}\left|\hat{r}_{j}^{i, k}(l)\right|$.

Moreover, the inverse of the m-sequence matrix can be used to determine the interference which is due to the $k^{\text {th }}$ user as specified in Eq. (10).

$$
\underline{\tilde{r}}_{j}^{i, k}=\left(M_{L}^{k}\right)^{-1} \cdot \underline{\underline{r}}_{j}^{i, k}
$$

$\underline{\tilde{r}}_{j}^{i, k}$ is the estimated signal of user $k$ from the received signal $\underline{r}_{j}^{i}$ at the $j^{\text {th }}$ sample of a chip. By doing the same procedure for $j=1 \ldots, m$, one can obtain an $(L \times m)$ matrix given by:

$$
\underline{R}^{i, k}=\left[\begin{array}{llll}
\widetilde{r}_{1}^{i, k} & \underline{\widetilde{r}}_{2}^{i, k} & \cdots & \underline{\widetilde{r}}_{m}^{i, k}
\end{array}\right]
$$

Each vector $\underline{\tilde{r}}_{j}^{i, k}$ is an estimate of the interference signal due to user $k$ at the $j^{\text {th }}$ sample of each chip during $i^{\text {th }}$ symbol duration. Knowing this estimate, the undesired $k^{\text {th }}$ user component can be removed from the total received signal (i.e. $\underline{r}^{i}$ ). Similarly, all undesired signals can be removed using the same process. The block diagram of the interference estimation is shown in Fig. 1. Note that the interference estimation process requires calculation of the inverse of $\mathrm{m}$ sequence matrix as appears in Eq. (10). The matrix inverse operation could be computationally expensive if the size of the matrix is large. To reduce the computational complexity, we propose using the Fast Walsh-Hadamard Transform. This will be further described in the next section.

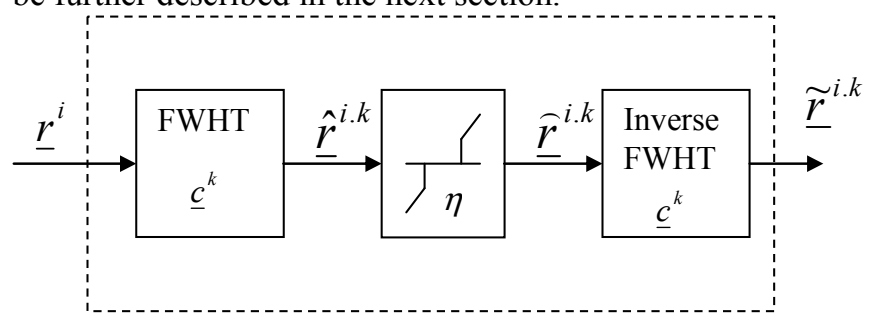

Fig. 1: Block diagram of Interference Estimation (I.E.) using FWHT

\section{CYCLIC CORRELATION COMPUTATION USING FAST WALSH-HADARMARD TRANSFORM}

Equations (7) and (10) involve an operation with the msequence matrix and its inverse, respectively. This section describes a low complexity algorithm for the m-sequence matrix operation through FWHT.

The relationship between the m-sequence matrix in Eq. (8) and the associated Hadamard matrix can be described by the following equation [8].

$$
M_{L}^{k}=V_{R} P_{w} H_{L+1} P_{S} V_{I}
$$

$V_{R}$ is a $(L \times L+1)$ matrix that reduces the size of the processed vector from $L+1$ to $L$; similarly, $V_{I}$ is a $(L+1 \times L)$ matrix that increases the size of the processed vector from $L$ to $L+1$. Both matrices require no special computation and are basically extensions of the identity matrix $I_{L}$ given by:

$$
\begin{aligned}
& V_{R}=\left[\begin{array}{ll}
0 & I_{L}
\end{array}\right] \\
& V_{I}=\left[\begin{array}{c}
0 \\
I_{L}
\end{array}\right]
\end{aligned}
$$

$P_{w}$ and $P_{s}$ are permutation matrices used for descrambling a vector. Therefore, their computational complexity is negligible. $H_{L+1}$ is a Walsh-Hadamard matrix with the size $(L+1 \times L+1)$. A Walsh-Hadamard matrix of a certain size can be generated recursively by the following methodology:

$$
\begin{aligned}
& H_{1}=[1] \\
& H_{i}=\left[\begin{array}{cc}
H_{i-1} & H_{i-1} \\
H_{i-1} & -H_{i-1}
\end{array}\right]
\end{aligned}
$$

Note that the dimension of these matrices is of the form $2^{j}$ where $j$ is an integer.

The correlation operation in Eq. (7) can be implemented by using Eq. (12) which only involves simple permutations and the FWHT. FWHT is similar to the Fast Fourier Transform, but only requires "addition" and "subtraction" operations. More details on FWHT can be found in [8]. The inverse of the m-sequence matrix in Eq. (7) is therefore obtained by the following equation.

$$
\left(M_{L}^{k}\right)^{-1}=V_{I}^{t} P_{s}^{t}\left(H_{L+1}\right)^{-1} P_{w}^{t} V_{R}^{t}
$$

The operation in Eq. (10) can also be performed using similar permutations and inverse FWHT. Fortunately, the inverse FWHT is similar to FWHT except for a constant factor of size $L+1$. In terms of computational complexity, the proposed PIC does not require any multiplication or division operations. The proposed technique only requires $m(L+1)\left(1+2 \cdot \log _{2}(L+1)\right)$ complex additions or subtractions 
per bit per interfering user, where $m$ is the number of samples per chip and $L$ is the number of chips (i.e. the length of the spreading code). In addition, the complexity grows linearly with the number of users as this technique can be implemented in parallel. Due to its complexity advantages, the FWHT provides an efficient and practical way to estimate the interference signal for interference cancellation.

\section{Simulation Results}

In this section, we present simulation results which are based on the proposed interference cancellation technique discussed earlier. The simulation scenario emphasizes on the near-far effect, where there is significant interference power from a nearby interferer. Three different channels (AWGN, slow Rayleigh fading and multipath) have been considered to obtain the results. The receiver's uncoded BER performance of the proposed PIC is compared with a conventional PIC technique [6] for each of these channels. The parameters of the simulations are assumed to be as follows: $L=31$ (i.e. spreading code length is 31) and $m=2$ (i.e. 2 samples per chip). Therefore, the processing gain will be around $15 \mathrm{~dB}$. To observe the effectiveness of interference cancellation techniques, two scenarios are considered for the signal-tointerference ratio i.e., $-10 \mathrm{~dB}$ (mild near-far effect) and $-20 \mathrm{~dB}$ (significant near-far effect).

Another parameter is the threshold $\eta$ in Eq. (9), which is usually difficult to be optimized. In general, the lower $\eta$ is chosen for low SNR or low SIR environments with a single dominant path or when it is desired to remove interference from the most dominant path in multipath-rich environments. To remove the interference from more number of paths, a higher $\eta$ may be used at the risk of increased inaccuracy in the estimation process. Figure 2 shows the block diagram of the receiver with the proposed interference cancellation technique.

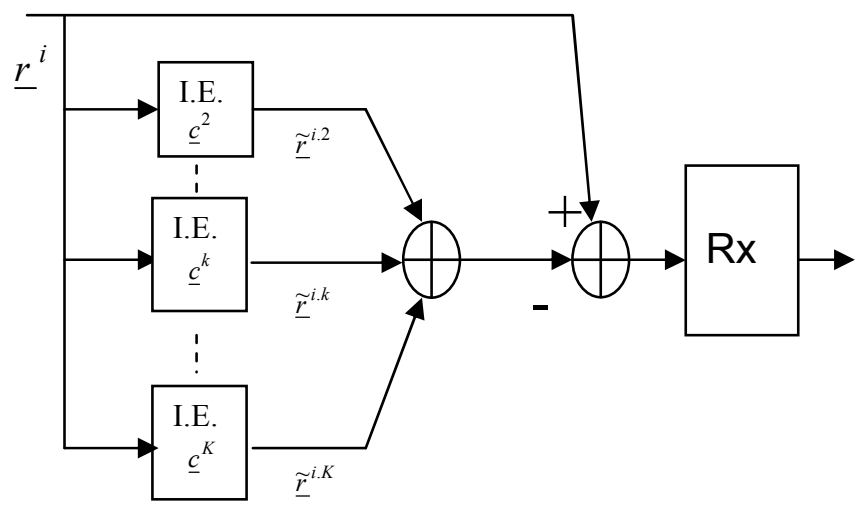

Fig. 2: Block diagram of proposed receiver with interference cancellation

Figure 3 displays uncoded BERs for an AWGN channel $(\eta=1.5)$. The proposed PIC matches the conventional PIC in the BER performance for both $\mathrm{SIR}=-10 \mathrm{~dB}$ and $\mathrm{SIR}=-20 \mathrm{~dB}$. But the conventional PIC requires perfect knowledge of the interference power level while the proposed scheme does not require this information. As expected, both conventional and proposed PICs outperform the system without any interference cancellation technique. The uncoded BER performance over a slow Rayleigh fading channel is shown in Fig. $4(\eta=1.5)$. Again, the performance of the proposed PIC matches the conventional PIC for both $\mathrm{SIR}=-10 \mathrm{~dB}$ and $\mathrm{SIR}=-20 \mathrm{~dB}$. However, the conventional PIC requires perfect information of actual signal fading amount in each symbol (fading statistics is not sufficient).

In summary, for the above single-path scenarios (AWGN and slow Rayleigh fading), the proposed PIC matches the conventional PIC in the BER performance. However, the conventional PIC requires accurate information about actual interference level or fading amount. In contrast, the proposed PIC does not require any such information.

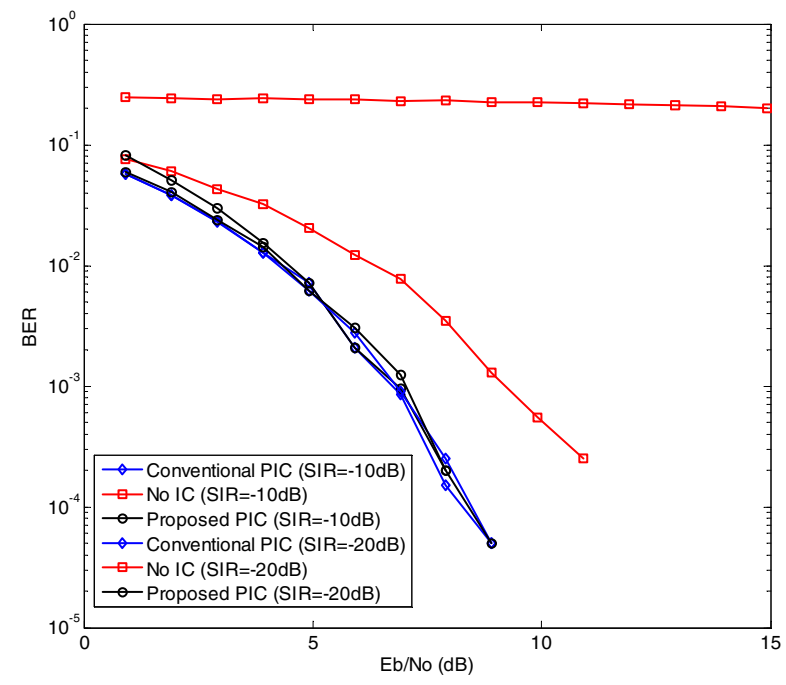

Fig. 3: Uncoded BER over an AWGN channel

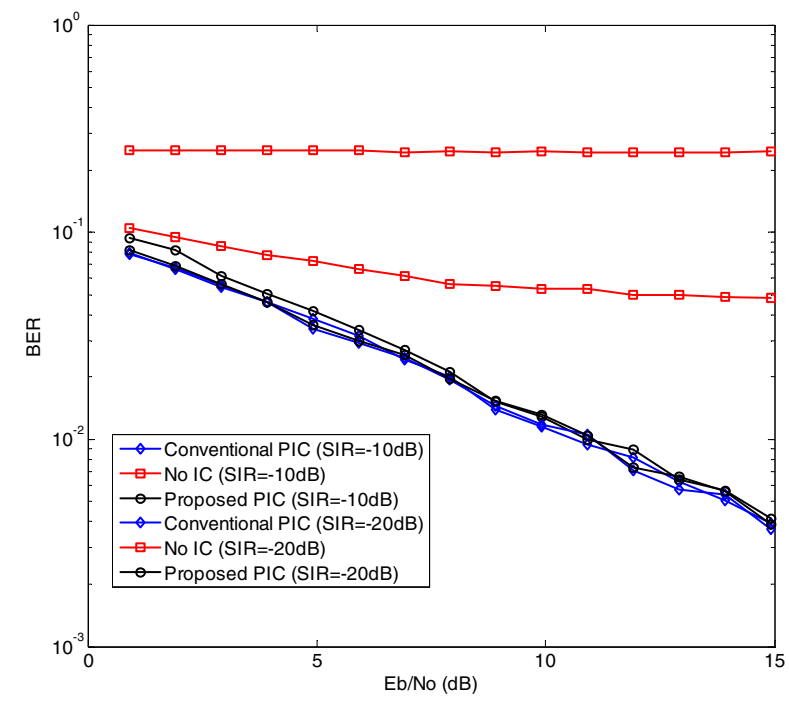

Fig. 4: Uncoded BER over a Rayleigh fading channel

For the multipath channel, we choose the channel impulse response to have the coefficients $\alpha_{0}(t)=1, \quad \alpha_{9}(t)=1 / 6$, and 
$\alpha_{19}(t)=-1 / 12$ for the desired user and the coefficients $\alpha_{0}(t)=1, \alpha_{4}(t)=-1 / 3, \alpha_{9}(t)=1 / 6$, and $\alpha_{19}(t)=-1 / 10$ for an interferer. The resulting BER performance for $\mathrm{SIR}=-10 \mathrm{~dB}$ (i.e. mild near-far effect) and $\eta=1.5$ is shown in Fig. 5. As observed, the proposed PIC has higher BER compared to the conventional PIC. This is only for the case when the conventional PIC has perfect knowledge of the channel conditions including interference power level and channel impulse response. Note that the channel conditions are usually very difficult to estimate. Also, as seen in Fig. 5, the proposed PIC matches the conventional PIC in the BER performance if the conventional PIC only has knowledge of the interference level (and no knowledge of channel impulse response).

Figure 6 shows the uncoded BER performance while SIR=$20 \mathrm{~dB}$ (i.e. significant near-far effect). For this case, the threshold has been chosen higher (i.e. $\eta=5$ ) in order to accurately estimate multipath interference. Similar to the previous case, the proposed PIC has higher BER compared to the conventional PIC that has perfect knowledge of channel conditions. However, the proposed PIC considerably outperforms the conventional PIC that only has knowledge of interference level (but no knowledge of channel impulse response). In other word, multipath interference significantly degrades the performance of the conventional PIC if it does not have any knowledge of channel impulse response. And its performance is similar to a system that does not employ any interference cancellation. On the other hand, our proposed PIC removes part of the multipath interference while channel conditions are unknown. If the channel conditions are also known, then the performance of the proposed PIC would match the conventional one in all cases.

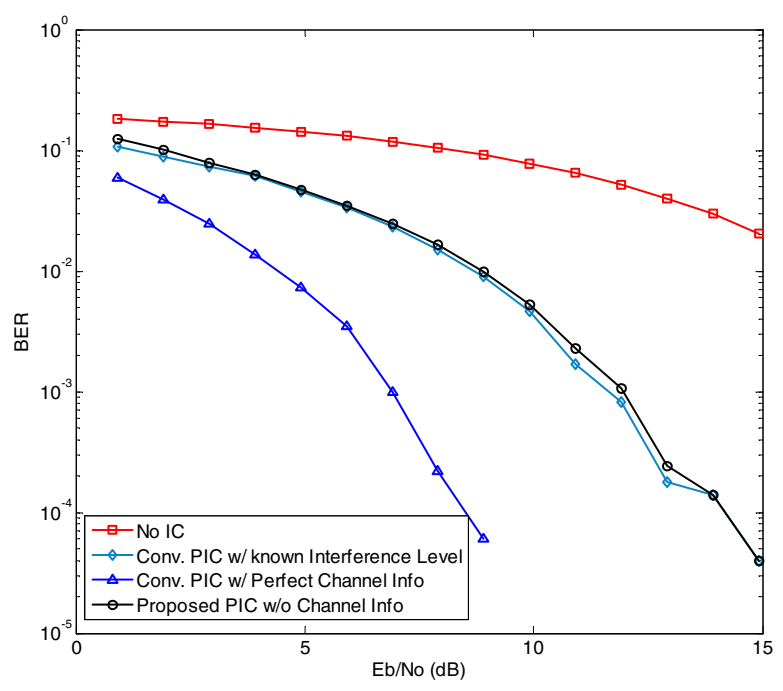

Fig. 5: Uncoded BER at $\mathrm{SIR}=-10 \mathrm{~dB}$ over a multi-path channel

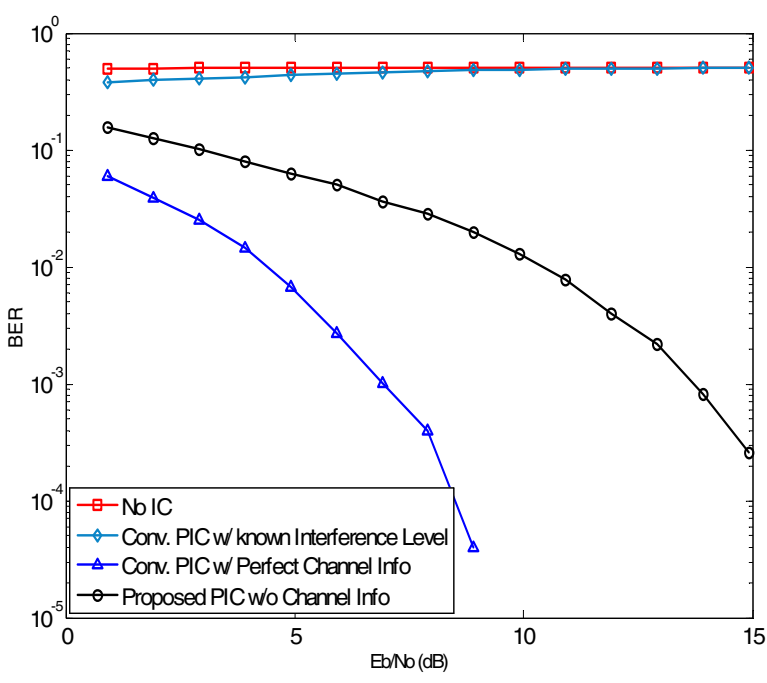

Fig. 6: Uncoded BER at $\mathrm{SIR}=-20 \mathrm{~dB}$ over a multipath channel

\section{CONCLUSIONS}

In this paper, we have proposed a low complexity parallel interference cancellation technique suitable for applications in wireless communication such as cellular, PCS or wireless local and personal area networks that are based on direct sequence code division multiple access. The proposed method does not require any knowledge of the interferer signal power level and its channel condition at the receiver. This property along with low implementation complexity makes this scheme suitable for fast fading environments where power control cannot be performed efficiently. A performance comparison between a conventional receiver (with and without interference cancellation) and a receiver with the proposed interference cancellation has been presented. The proposed technique demonstrates considerable improvement, particularly for multipath scenarios where significant near-far effect exists.

\section{REFERENCES}

[1] T. C. Yang and W.-B. Yang, "Interference Suppression for codedivision multiple-access communications in an underwater acoustic channel," J. Acoust. Soc. Am, 126(1), 220-228, July 2009.

[2] R. Lupas and S. Verd, "Near-far resistance of multiuser detectors in asynchronous channels," IEEE Trans. Commun., vol. 38, pp. 496-508, Apr. 1990.

[3] _L "Linear multiuser detector in synchronous CDMA channels," IEEE Trans. Inform. Theory, vol. 35, pp. 123-136, Jan. 1989.

[4] R. Fantacci, "Proposal of an interference cancellation receiver with low complexity for DS/CDMA mobile communication systems," IEEE Trans. Vehicular Technology, vol. 48, pp.1039-1046, July 1999.

[5] P. Patel and J. Holtzman, "Analysis of a simple successive interference cancellation scheme in a DS/CDMA system," IEEE J. Select. Areas Commun., vol. 12, pp. 796-807, June 1994.

[6] A. Duell-Hallen, "A family of multiuser decision-feedback detectors for asynchronous CDMA channels," IEEE Trans. Commun., vol. 43, pp. 421-434, Feb./Mar./Apr. 1995.

[7] Cohn and Lempel, "On Fast M-sequence Transforms," IEEE Trans. Information Theory, Vol. 23, pp. 135-137, 1977.

[8] Henry S. Chang, "Detection of weak, broadband signals under Dopplerscaled, multipath propagation", PhD Thesis, University of Michigan (1992). 